\title{
IDENTIFICAÇÃO DE CARGAS LINEARES E NÃO-LINEARES EM SISTEMAS ELÉTRICOS RESIDENCIAIS USANDO TÉCNICAS PARA SELEÇÃO DE ATRIBUTOS E REDES NEURAIS ARTIFICIAIS
}

\author{
Ricardo A. S. Fernandes*
}

\author{
Ivan N. da Silva*
}

\author{
Mário Oleskovicz* \\ *USP / EESC / SEL \\ Av. Trabalhador Sancarlense, 400 Centro, CEP 13566-590 \\ São Carlos - SP
}

\section{RESUMO}

Este trabalho consiste em apresentar um método para a identificação de cargas lineares e não-lineares comumente encontradas em sistemas elétricos residenciais. Desta identificação, soluções viáveis poderão ser aplicadas com o intuito de mitigar os níveis de emissão das correntes harmônicas geradas, advindas principalmente por cargas com características não-lineares. No desenvolvimento do método, utilizaram-se de técnicas para a seleção de atributos, de forma a minimizar a dificuldade em se identificar as cargas conectadas ao sistema. A etapa posterior de identificação foi realizada pela aplicação de redes neurais artificiais. Todas as situações de distorção harmônica foram geradas em laboratório por uma fonte de alimentação, onde em sua saída foram alocados analisadores de energia, responsáveis pela extração das medidas necessárias sobre as cargas residenciais em análise. Os resultados obtidos foram considerados satisfatórios, mostrandose que a metodologia proposta pode ser também empregada pelas concessionárias de energia elétrica para que estas obtenham informações sobre o perfil das cargas instaladas em consumidores residenciais.

PALAVRAS-CHAVE: Identificação de cargas lineares e nãolineares, componentes harmônicas, redes neurais artificiais.

\begin{abstract}
Identification of Harmonic Current Sources in SinglePhase Power Systems Using Feature Selection Techniques and Artificial Neural Networks

This work presents a method to identify linear and nonlinear loads commonly encountered in residential electrical systems. From this method, feasible solutions can be applied to mitigate the high levels of harmonic currents, generated mainly by nonlinear loads. Techniques of feature selection were used to data preprocessing and to minimize the effort in identification of loads connected to the electrical system. For the next step, the load identification, artificial neural networks were applied. All harmonic distortion situations were created in laboratory from a power source, and in its outputs were inserted the loads and power quality analyzers, which perform the extraction of all measurements. The obtained results were considered satisfactory, which show that the methodology can be employed by power distribution companies in order to obtain information about the profile of loads used by residential consumers.
\end{abstract}

KEYWORDS: Identification of linear and nonlinear loads, harmonic components, artificial neural networks. 


\section{INTRODUÇÃO}

Com as instalações residenciais utilizando cada vez mais cargas não-lineares como computadores, televisores, refrigeradores, condicionadores de ar, circuitos de iluminação, dentre outros, nota-se um aumento dos níveis das correntes harmônicas encontradas nos sistemas elétricos, os quais podem contribuir para a degradação da Qualidade da Energia Elétrica (QEE). Apenas como fato ilustrativo, tem-se hoje a preocupação com o uso racional da energia, onde em certos casos verifica-se a substituição de cargas praticamente lineares (alto consumo) por cargas não-lineares (baixo consumo). Como exemplo, pode-se citar a substituição de lâmpadas incandescentes por lâmpadas fluorescentes compactas.

No entanto, observando o sistema elétrico como um todo, mesmo com a proliferação de cargas não-lineares, constatarse-á que as residências possuem uma distorção harmônica muitas vezes aceitável; porém, a soma das distorções harmônicas geradas pelos consumidores residenciais de um determinado alimentador pode contribuir para a amplificação da distorção harmônica total deste alimentador, quando somadas às distorções ocasionadas pelos consumidores de grande porte. Assim, algumas correntes harmônicas podem também apresentar amplitudes além do esperado e, por consequiência, causar diversos problemas relacionados à QEE (Bezerra et al., 2001). Como resultado da amplificação dos níveis de harmônicas, tem-se a má qualidade da energia que pode ser refletida para as concessionárias, consumidores próximos à fonte geradora de harmônicas e ao próprio estabelecimento gerador. Muitas das vezes, os próprios consumidores são os grandes prejudicados, devido à maior sensibilidade de suas cargas (Dugan et al., 2004).

Neste contexto, caso não haja meios específicos de controle, qualquer sistema com grande quantidade de cargas não-lineares estará vulnerável a problemas refletidos na qualidade da energia. Desta situação, poder-se-ia evidenciar um mau funcionamento de equipamentos, baixo fator de potência, distorção na forma de onda da tensão e/ou corrente, aquecimento de cabos, dentre outros (Dugan et al., 2004).

Devido aos problemas apontados anteriormente, muitas das pesquisas são realizadas com relação à identificação e controle de harmônicas. Assim, tais pesquisas encontram-se divididas, como denominado por Kumar et al. (2004), em análise direta de harmônicas e análise reversa de harmônicas. Cabe comentar que, além destas duas frentes de pesquisa, existem também os métodos de detecção e classificação dos distúrbios acarretados com a má qualidade da energia; no entanto, estes geralmente não se concentram apenas nas distorções harmônicas (Ferreira et al., 2009).

Dentre os estudos que compreendem a análise direta de harmônicas, grande parte destina-se às técnicas para extração de harmônicas, normalmente baseados em ferramentas matemáticas como as Transformadas de Fourier e as Transformadas Wavelet (Ng et al., 2005; Tse, 2006; Limin et al., 2007; Tarasiuk, 2007; Pádua et al., 2007); monitoração de tensões e correntes harmônicas aplicando as principais normas e/ou recomendações (Prudenzi et al., 2001; Halpin, 2005; Lowe et al., 2006; Maitra et al., 2007); desenvolvimento de filtros para a mitigação das componentes harmônicas que apresentem níveis elevados (Czarnecki, 2000; Pomilio e Deckmann, 2007; Quesada et al., 2007); e modelagem de cargas nãolineares para que possam ser utilizadas em simulações de sistemas elétricos de baixa, média, alta ou extra-alta tensão (Acarkan e Erkan, 2007; Mota et al., 2004).

No entanto, durante a última década, as pesquisas estão mais voltadas aos métodos de análise reversa de harmônicas, pois estes são responsáveis por estimar harmônicos em barramentos, calcular o fluxo de potência das harmônicas (Variz et al., 2008), além de realizar tarefas como a localização e identificação de cargas que absorvam elevadas correntes harmônicas, seja em barramentos dos sistemas de distribuição das concessionárias, em plantas industriais e até mesmo em consumidores residenciais (Srinivasan et al., 2006; Fernandes et al., 2008). É neste contexto que o presente trabalho se insere, em que se realiza a identificação de cargas lineares e não-lineares em sistemas elétricos residenciais.

Os primeiros métodos de análise reversa foram propostos por Heydt (1989), em que se realizava uma estimação de harmônicas sobre os barramentos que não possuíam medidores, para assim determinar quais se encontravam sob a influência de altos níveis de distorção harmônica. Porém, até então não se dava tanta importância às correntes harmônicas demandadas por sistemas industriais, comerciais e residenciais, devido às cargas não-lineares ainda não terem se proliferado. Conseqüentemente, os primeiros métodos desenvolvidos apenas conseguiam identificar uma carga por vez. Assim, Varadan e Makram (1996) propuseram um método baseado em mínimos quadrados que estimava o estado das harmônicas nos barramentos e, por meio de uma análise feita sobre os dados obtidos, tal método era capaz de identificar a classe de cargas que provavelmente encontrava-se conectada ao barramento. Para tanto, houve a necessidade de determinar três classes de cargas, isto porque as mesmas possuíam características particulares, sendo que a classe " $A$ " tinha predominância das harmônicas de $5^{a}, 7^{a}, 11^{a}$ e $13^{a}$ ordens; a classe "B" era determinada por cargas com maior predominância de harmônicas de $3^{a}, 5^{a}$ e $7^{a}$ ordens; e a classe "C" constituída por cargas com harmônicas predominantes de $3^{a}$ e $5^{a}$ ordens.

Já em Kumar et al. (2004), um método para identificação de cargas não-lineares em barramentos é proposto, no qual duas etapas são realizadas. Na primeira etapa são encontrados os 
possíveis barramentos onde estão conectadas as cargas nãolineares, usando-se aqui um método de estimação baseado em mínimos quadrados. A segunda etapa elege um dos barramentos selecionados pela primeira etapa, empregando-se então no mesmo um método baseado na norma Euclidiana que visa identificar as cargas não-lineares mais expressivas.

Observa-se que muitas pesquisas até então utilizavam técnicas convencionais, como o próprio método dos mínimos quadrados, para a estimação de estado das harmônicas em barramentos, onde posteriormente as cargas não-lineares eram identificadas, sendo que estas representavam barramentos ou uma classe de cargas e não as cargas propriamente ditas. Entretanto, além dos métodos convencionais, em alguns trabalhos anteriores, já haviam sido aplicados sistemas inteligentes como as Redes Neurais Artificiais (RNAs), em que algumas de suas vantagens e desvantagens foram apresentadas. Um dos trabalhos pioneiros foi realizado por Varadan e Makram (1994), em que se empregou RNAs com arquitetura de múltiplas camadas (MLP - Multilayer Peceptron), sendo as mesmas treinadas com o algoritmo backpropagation. A partir deste estudo, a aplicação de sistemas inteligentes para a tarefa de identificação de cargas não-lineares se proliferou, onde tanto RNAs (Chan et al., 2000; Niekerk et al., 2002; Lin et al., 2005) como sistemas de inferência fuzzy (Nawi et al., 2003; Ferrero et al., 2007) passaram a ser utilizados devido à apresentação de resultados satisfatórios.

Ainda com relação ao trabalho desenvolvido por Varadan e Makram (1994), o mesmo fez uso de uma RNA treinada para identificar as cargas conforme as classes determinadas pelo IEEE Std. 519-1992. No referido trabalho, as cargas foram separadas em lineares e não-lineares de acordo com o ângulo de fase das correntes, em que se demonstrou que o ângulo é um atributo irrelevante para tarefas de identificação de cargas não-lineares, pois não se consegue caracterizar distintos tipos de cargas não-lineares por meio desta variável.

Na proposta de Niekerk et al. (2002) foi também utilizada uma rede neural do tipo $M L P$, cujos algoritmos de treinamento foram variados entre bayesian regularisation e resilient backpropagation (Demuth et al., 2007). Tal pesquisa possui grande semelhança àquela relatada nos dois parágrafos anteriores (Varadan e Makram, 1994) devido a também classificar as cargas conforme regido pelo IEEE Std. 5191992.

Já em Nawi et al. (2003), os sistemas fuzzy foram aplicados com o intuito de se identificar cargas industriais como inversores de freqüência, controladores de velocidade para máquinas elétricas, além de fornos a arco. As cargas foram identificadas por suas correntes harmônicas de $3^{a}, 5^{a}, 7^{a}, 9^{a}$ e $11^{a}$ ordens, sendo as regras lingüísticas do sistema fuzzy determinadas conforme o nível de cada uma destas correntes harmônicas.

Pesquisas mais recentes ainda trabalham com sistemas de distribuição e sistemas elétricos industriais. Porém, começam a crescer os estudos relacionados à identificação de cargas não-lineares em sistemas elétricos residenciais. Dentro deste contexto, Umeh e Mohamed (2005) propuseram um sistema fuzzy com regras lingüísticas baseadas nos níveis percentuais da Distorção Harmônica Total (DHT) de corrente.

Já Srinivasan et al. (2006) propõem um método a partir da aplicação da Transformada Rápida de Fourier (TRF) para a extração do espectro de freqüências das correntes, considerando-se o ângulo de fase e a amplitude apenas das componentes de ordem ímpar até a $15^{a}$ ordem, resultando assim em 16 variáveis que foram fornecidas como entradas às RNAs implementadas. Neste mesmo trabalho, modelos baseados em RNAs (MLP - Multilayer Perceptron, RBF Radial Basis Function e SVM - Support Vector Machines) foram utilizados para a identificação das cargas presentes no sistema elétrico residencial criado em laboratório.

Seguindo o contexto apresentado por Srinivasan et al. (2006), a pesquisa aqui proposta também realiza a identificação de cargas conectadas em sistemas elétricos residenciais; porém, com o intuito de mostrar que, assim como em Varadan e Makram (1994), o ângulo de fase é um atributo irrelevante para a identificação de cargas não-lineares. Vale ressaltar que, diferentemente do trabalho de Srinivasan et al. (2006), foram adquiridas componentes harmônicas pares e ímpares até a $25^{a}$ ordem e que empregaram-se técnicas para a seleção dos atributos mais relevantes, as quais proporcionam a redução do número de correntes harmônicas utilizadas para a tarefa de identificação das cargas presentes em um sistema elétrico residencial. Desta forma, obtiveram-se como variáveis de entrada apenas a amplitude de 6 das 25 correntes analisadas, constatando assim, grande redução na quantidade de variáveis de entrada fornecidas às RNAs quando comparado ao trabalho desenvolvido por Srinivasan et al. (2006), o qual não empregou nenhuma técnica para selecionar atributos. Consequientemente, esta pesquisa também tem como atrativo salientar que a utilização de técnicas de seleção de atributos contribui de maneira significativa e efetiva para o processo de escolha das variáveis mais relevantes ao sistema a ser mapeado pela abordagem neural.

Sendo assim, o principal objetivo deste trabalho é gerar uma sistemática que possa ser aplicada à identificação dos perfis de cargas instalados em residências, com o intuito de fornecer às concessionárias de energia elétrica uma informação útil à monitoração da QEE. Além do mais, esta informação poderá ser utilizada para se realizar cálculos de fluxo de potência harmônico em sistemas de distribuição, visto que até então não há a possibilidade de garantir boa precisão para 
este cálculo, justamente devido à falta de informações relacionadas às componentes harmônicas características das cargas utilizadas pelos consumidores finais de energia.

\section{METODOLOGIA PROPOSTA}

Apresenta-se nesta seção uma visão geral da metodologia proposta, a qual se encontra ilustrada pela Figura 1.

$\mathrm{O}$ que se refere aos aspectos físicos do sistema, estes serão abordados na Seção 3. Entretanto, cabe comentar que todo o procedimento experimental foi baseado na fonte de alimentação, em que foram geradas 14 formas de onda distintas, sendo que 9 delas foram configuradas com distorções harmônicas (DHTs de respectivamente 0,94\%; 1,51\%; $1,94 \% ; 2,62 \% ; 3,02 \% ; 3,42 \% ; 3,94 \% ; 4,42 \%$ e $5 \%$ ) dentro dos limites estabelecidos pelo IEEE Std. 519-1992. Outras 4 foram configuradas com distorções acima destes limites e outra forma de onda foi configurada em condições ideais (sem distorções harmônicas). No entanto, todas as formas de onda distorcidas tiveram como base a forma de onda ideal, a qual foi configurada com tensão em $127 \mathrm{~V}$ e ângulo de fase em $90^{\circ}$.

Cabe comentar que, em conformidade com o IEEE Std. 5191992 , todas as formas de onda distorcidas foram configuradas com até 5\% de DHT de tensão e um valor máximo de 3\% de Distorção Harmônica Individual (DHI) de tensão.

Por meio das Figuras de 2 a 5 podem ser visualizadas as for-

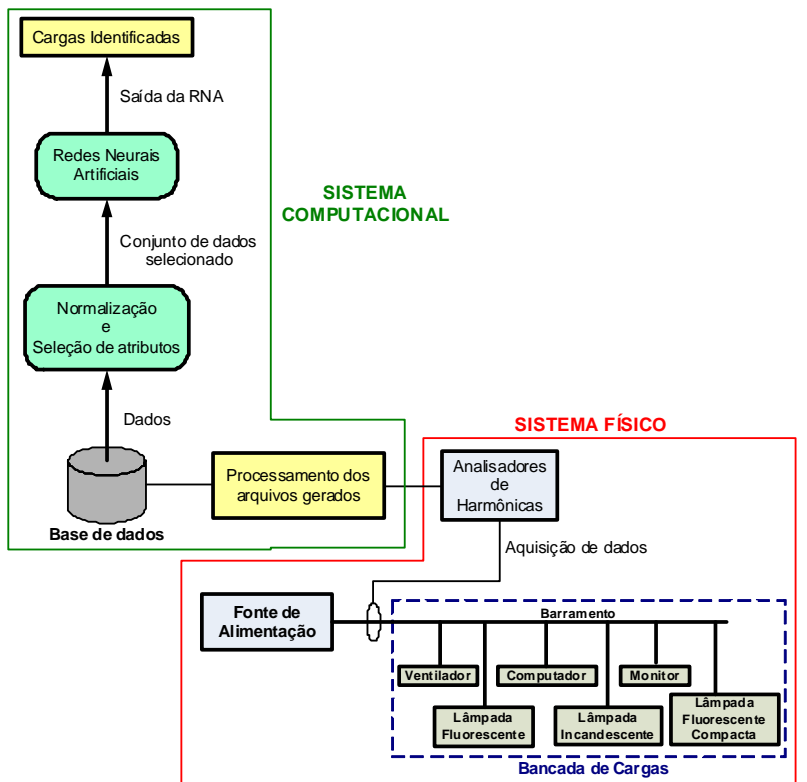

Figura 1: Diagrama de blocos denotando uma visão geral da metodologia proposta.

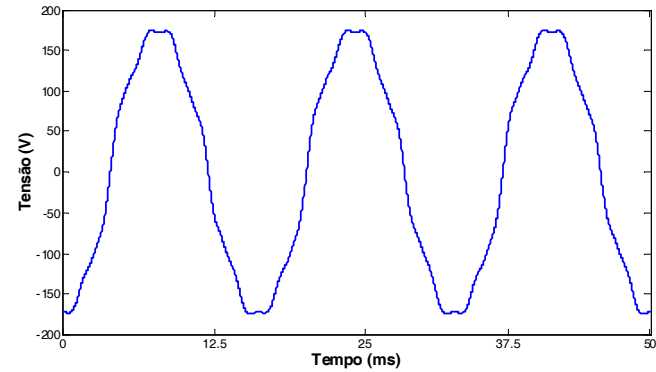

Figura 2: Forma de onda de tensão com uma DHT de $8,12 \%$.

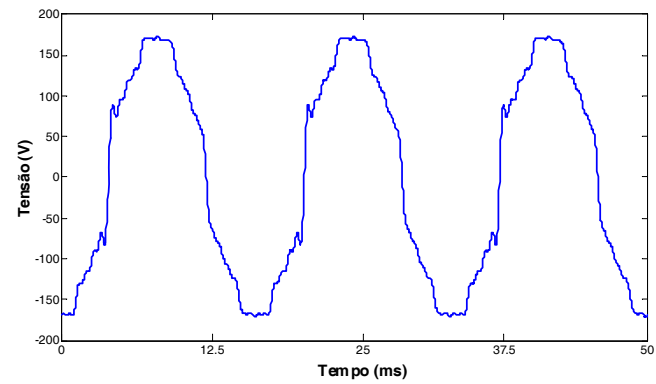

Figura 3: Forma de onda de tensão com uma DHT de $14,52 \%$.

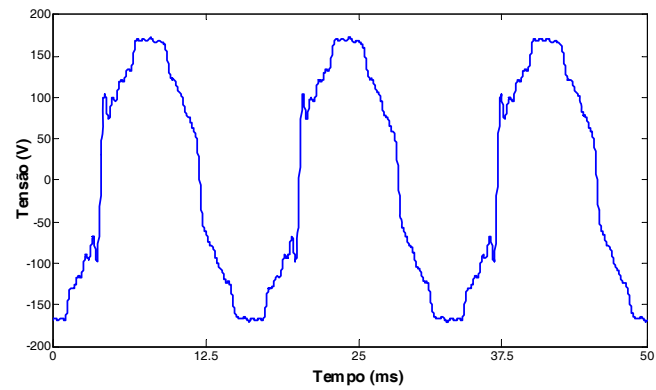

Figura 4: Forma de onda de tensão com uma DHT de $16,34 \%$.

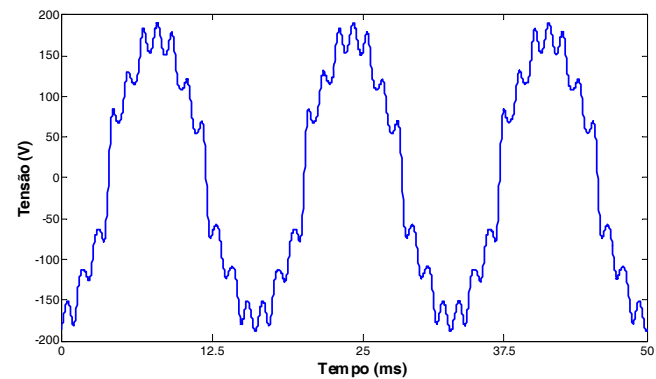

Figura 5: Forma de onda de tensão com uma DHT de $16,41 \%$.

mas de onda distorcidas, as quais extrapolam os limites préestabelecidos. 
Já o quadro de cargas foi projetado de modo a permitir o acionamento individual ou até mesmo combinações entre as cargas. Assim, para todas as 6 cargas, teremos $64\left(2^{6}\right)$ possíveis combinações ou medições. Ressalta-se que uma das combinações leva em consideração que nenhuma carga se encontra conectada ao sistema. Portanto, foram possíveis 63 medições com cargas.

Além das formas de onda distorcidas, outras 189 medidas aleatórias foram realizadas variando-se a amplitude da tensão de saída da fonte entre $\pm 15 \%$. No entanto, para cada uma destas 189 situações geradas, dois analisadores de energia distintos foram empregados (Fluke $囚$ e California Instruments, os quais serão posteriormente apresentados na Seção 3 ), permitindo que o número de medidas dobre, chegando-se então a um total de 378 aquisições. A utilização de analisadores de energia distintos garantiu que as assinaturas harmônicas obtidas fossem diferentes em poucos níveis percentuais. Tais diferenças advêm das características intrínsecas e construtivas de ambos os equipamentos (hardware e software), sendo os erros de medição em corrente de $\pm 0,05 \mathrm{~A}$ para o equipamento da Fluke $\mathbb{R} \pm \pm 0,01 \mathrm{~A}$ para o California

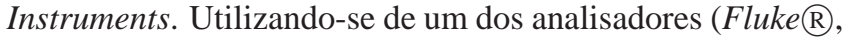
Seção 3), ainda foram extraídas outras 126 medidas aleatórias sob as cargas alimentadas com formas de onda que foram distorcidas seguindo os limites estabelecidos pelo IEEE Std. 519-1992.

Desta forma, por meio do analisador da California Instruments, obteve-se um total de 1071 medições, que correspondem à soma das aquisições realizadas para as cargas alimentadas com as 13 formas de onda distorcidas, considerandose também aquelas que extrapolam os limites de distorções $(63 \times 13=819)$; às aquisições realizadas com as cargas sendo alimentadas pela forma de onda sem distorção (63); e pelas aquisições realizadas quando se variou a amplitude da tensão de saída da fonte entre $\pm 15 \%$ (189). Já pelo analisador da Fluke, obteve-se um total de 315 medidas, que correspondem às 126 aquisições referentes às cargas alimentadas por formas de onda distorcidas e 189 medidas realizadas a partir da variação da amplitude de tensão entre $\pm 15 \%$. Portanto, a base de dados é constituída por um total de 1386 medições.

Vale ressaltar que todas as medidas foram realizadas com as cargas em regime permanente, conforme estabelecido pelo IEEE Std. 519-1992, para que a real assinatura de uma carga possa ser extraída.

Afirma-se que os dados representativos das assinaturas harmônicas, ou das cargas individuais e/ou da combinação entre as mesmas, continham apenas correntes harmônicas da $1^{a}$ a $25^{a}$ ordem com suas respectivas amplitudes. O ângulo de fase não foi considerado devido à pesquisa de Varadan e Makram (1994) mostrar que este não é um atributo relevante para a identificação de cargas não-lineares. Portanto, para cada medição foram extraídas 25 variáveis.

No que segue, são apresentadas por meio da Tabela 1, as amplitudes das correntes harmônicas individuais de cada uma das cargas quando alimentadas por tensão sem distorção.

Tabela 1: Assinatura harmônica individual de corrente das cargas sob análise.

\begin{tabular}{|c|c|c|c|c|c|c|}
\hline $\begin{array}{c}\text { Ordem } \\
\text { Harmô- } \\
\text { nica }\end{array}$ & PC & MON & LFC & LF & LI & VE \\
\hline $1^{a}$ & 0,72 & 0,41 & 0,21 & 0,28 & 0,79 & 1,28 \\
\hline $2^{a}$ & 0,66 & 0,38 & 0,17 & 0,22 & 0 & 0,01 \\
\hline $3^{a}$ & 0,56 & 0,33 & 0,1 & 0,13 & 0 & 0 \\
\hline $4^{a}$ & 0,44 & 0,27 & 0,05 & 0,07 & 0 & 0 \\
\hline $5^{a}$ & 0,3 & 0,2 & 0,04 & 0,05 & 0 & 0 \\
\hline $6^{a}$ & 0,18 & 0,13 & 0,04 & 0,05 & 0 & 0 \\
\hline $7^{a}$ & 0,09 & 0,07 & 0,03 & 0,04 & 0 & 0 \\
\hline $8^{a}$ & 0,06 & 0,03 & 0,02 & 0,03 & 0 & 0 \\
\hline $9^{a}$ & 0,07 & 0,02 & 0,02 & 0,03 & 0 & 0 \\
\hline $10^{a}$ & 0,06 & 0,02 & 0,02 & 0,03 & 0 & 0 \\
\hline $11^{a}$ & 0,05 & 0,02 & 0,02 & 0,02 & 0 & 0 \\
\hline $12^{a}$ & 0,02 & 0,02 & 0,02 & 0,02 & 0 & 0 \\
\hline $13^{a}$ & 0,01 & 0,01 & 0,02 & 0,02 & 0 & 0 \\
\hline $14^{a}$ & 0,01 & 0 & 0,01 & 0,02 & 0 & 0 \\
\hline $15^{a}$ & 0,01 & 0 & 0,01 & 0,02 & 0 & 0 \\
\hline $16^{a}$ & 0,01 & 0,01 & 0,01 & 0,02 & 0 & 0 \\
\hline $17^{a}$ & 0 & 0 & 0,01 & 0,02 & 0 & 0 \\
\hline $18^{a}$ & 0 & 0 & 0,01 & 0,01 & 0 & 0 \\
\hline $19^{a}$ & 0 & 0 & 0,01 & 0,01 & 0 & 0 \\
\hline $20^{a}$ & 0 & 0 & 0,01 & 0,01 & 0 & 0 \\
\hline $21^{a}$ & 0 & 0 & 0,01 & 0,01 & 0 & 0 \\
\hline $22^{a}$ & 0 & 0 & 0,01 & 0,01 & 0 & 0 \\
\hline $23^{a}$ & 0 & 0 & 0,01 & 0,01 & 0 & 0 \\
\hline $24^{a}$ & 0 & 0 & 0,01 & 0,01 & 0 & 0 \\
\hline $25^{a}$ & 0 & 0 & 0,01 & 0,01 & 0 & 0 \\
\hline & & & & & & \\
\hline
\end{tabular}

Para uma melhor adequação da tabela, utilizaram-se os seguintes rótulos para se referir a cada uma das cargas: PC (computador pessoal), MON (monitor), LFC (lâmpada fluorescente compacta), LF (lâmpada fluorescente acionada por reator eletrônico), LI (lâmpada incandescente) e VE (ventilador).

Além das assinaturas harmônicas de corrente das cargas, são apresentadas por meio das Figuras de 6 a 11 as formas de onda de corrente de cada carga quando da alimentação destas por um sinal puramente senoidal (sem distorção harmônica).

Apenas como caráter explicativo, cabe comentar que, para manter a uniformidade da base de dados, alguns módulos de software foram desenvolvidos por meio da ferramenta computacional Matlab $囚$, de forma a se realizar a adequação dos arquivos (extração dos cabeçalhos) provenientes dos analisa- 


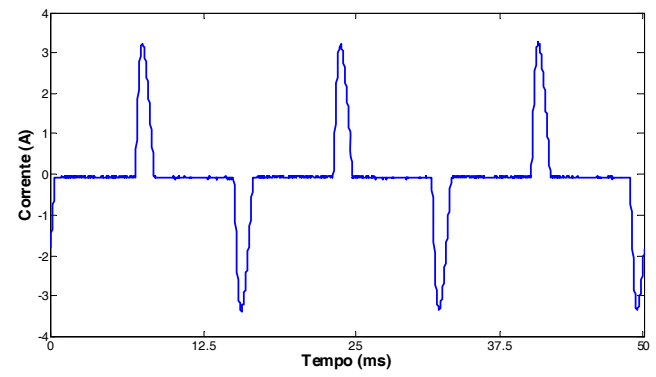

Figura 6: Forma de onda da corrente quando da alimentação de um PC (Computador Pessoal).

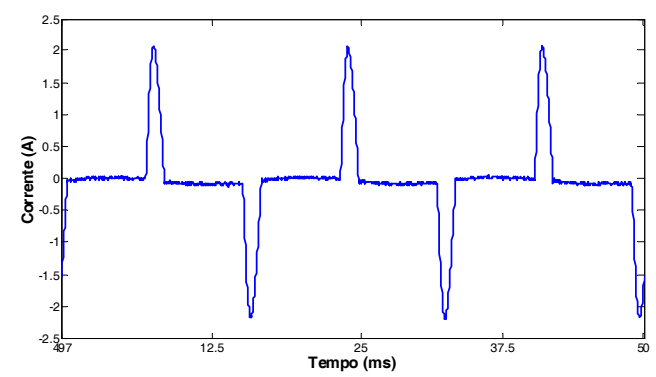

Figura 7: Forma de onda da corrente quando da alimentação de um Monitor (MON).

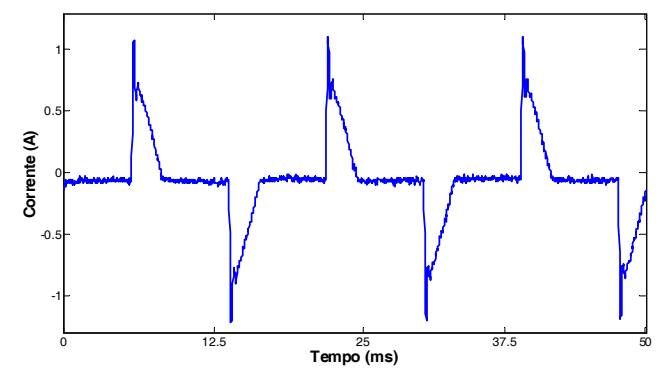

Figura 8: Forma de onda da corrente quando da alimentação de uma Lâmpada Fluorescente Compacta (LFC).

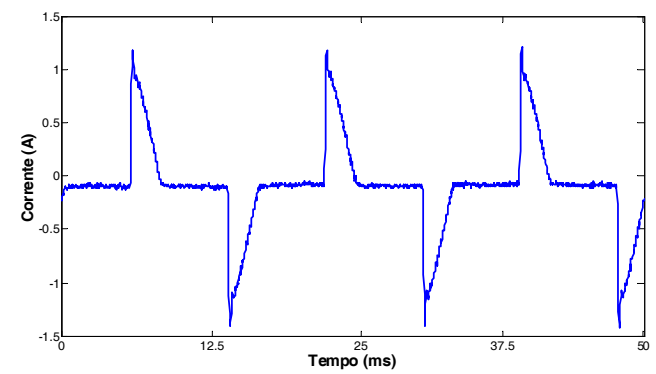

Figura 9: Forma de onda da corrente quando da alimentação de uma Lâmpada Fluorescente com reator eletrônico (LF).

dores de energia.

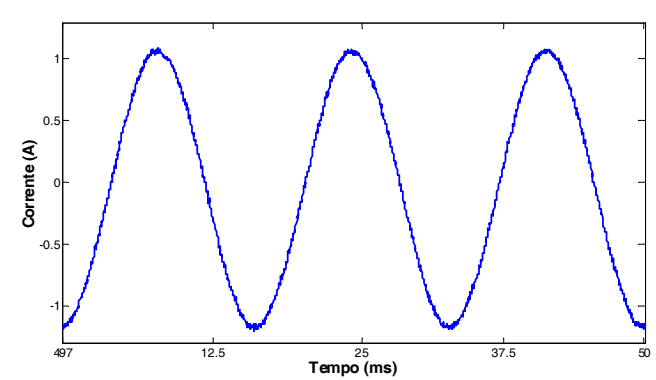

Figura 10: Forma de onda da corrente quando da alimentação de uma Lâmpada Incandescente (LI).

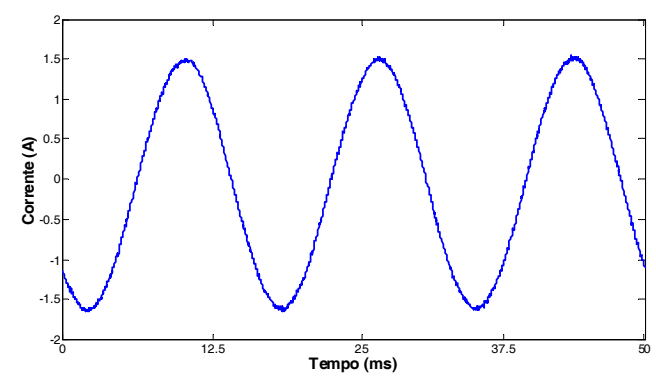

Figura 11: Forma de onda da corrente quando da alimentação de um Ventilador (VE).

Tendo em mãos os arquivos totalmente adequados, a base de dados pôde então ser gerada, a qual possuía 1386 instâncias, contendo cada uma delas um total de 25 atributos.

Devido à grande quantidade de atributos na base de dados, o treinamento das RNAs tornou-se uma tarefa de alto custo computacional. Isto decorre do fato de que muitos atributos não contribuem efetivamente para a extração dos padrões de assinaturas harmônicas. Em face disto, o emprego do seletor de atributos foi de extrema necessidade. Sendo assim, foi verificado o desempenho dos três métodos para seleção de atributos, os quais serão explicitados na Seção 4. Cabe adiantar que o desempenho de cada um desses métodos foi avaliado quanto ao nível de relevância dos atributos. Entretanto, apenas como caráter informativo e ilustrativo, foi obtido o tempo gasto por cada um destes métodos. Ressalta-se que os seletores de atributos são executados somente na fase de projeto da metodologia proposta e, portanto, este tempo não é relevante à identificação dos perfis de carga. Os resultados obtidos por estes métodos serão apresentados e discutidos na Seção 4.

Com os atributos selecionados, passou-se então a utilizar uma nova base de dados constituída apenas pelos atributos mais relevantes à identificação das cargas. Assim, a nova base gerada pôde ser dividida em dois conjuntos, os quais foram utilizados nos processos de treinamento e validação das RNAs. 
O conjunto de treinamento foi, por conseguinte, submetido às RNAs, as quais foram treinadas por meio do algoritmo de Levenberg-Marquardt para que pudessem extrair os padrões de assinaturas harmônicas das cargas. Para tanto, seis RNAs foram empregadas, sendo que cada RNA tornouse especialista na identificação de um determinado equipamento. Esta estratégia fez com que o desempenho na fase de identificação das cargas fosse ainda melhor. Ressalta-se que as RNAs foram empregadas aqui justamente pelo fato de conseguirem mapear eficientemente a dinâmica comportamental do sistema analisado, garantindo-se boa classificação mesmo quando os sinais de entrada sofrerem pequenos desvios (inerentes a equipamentos de fabricantes diferentes), pois a mesma possui capacidade de generalização de soluções, e que implica em potencial para também ser tolerante a essas perturbações (falhas) de baixo impacto. Todos os aspectos relacionados às RNAs serão abordados com maior profundidade na Seção 5.

\section{ASPECTOS DA BANCADA DE ENSAIOS}

A bancada experimental foi basicamente composta por uma fonte de alimentação, dois analisadores de energia, um quadro de cargas e um microcomputador responsável pelo armazenamento dos dados e pela execução do sistema de identificação das cargas conectadas ao sistema elétrico. Para uma melhor compreensão desta bancada de ensaios, um diagrama de blocos da mesma pode ser visualizado por meio da Figura 12.

A princípio, empregou-se um total de seis cargas dentre lineares e não-lineares. O quadro de cargas foi alimentado com uma forma de onda puramente senoidal a fim de que pudessem ser extraídas as assinaturas harmônicas reais de cada carga, bem como de suas respectivas combinações. No entanto, conforme apresentado na seção anterior, também foram criadas mais 9 formas de onda com um conteúdo harmônico dentro dos limites estabelecidos pela recomendação IEEE Std. 519-1992, além de 4 formas de onda com conteúdo harmônico extrapolando estes limites, a fim de melhor

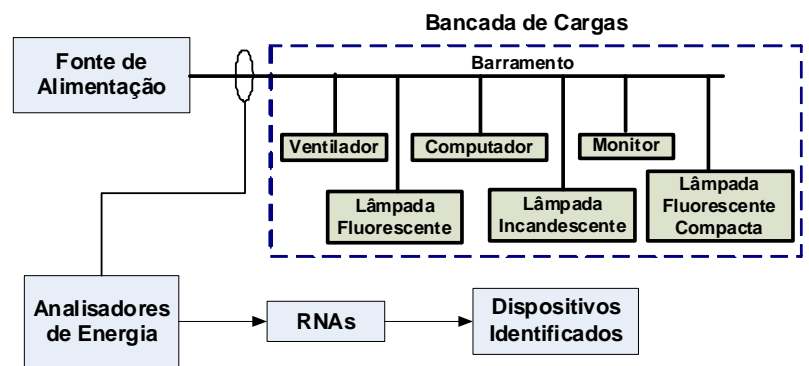

Figura 12: Diagrama de blocos representando a bancada experimental utilizada nos ensaios laboratoriais. representar um sistema elétrico residencial.

As assinaturas harmônicas foram então extraídas com o auxílio de dois analisadores de energia que serão comentados mais adiante.

\subsection{Fonte de Alimentação}

Para a alimentação das cargas foi utilizada uma fonte monofásica, modelo 5001iX da California Instruments (California Instruments, 2007), com potência de $5 \mathrm{kVA}$. Esta fonte de alimentação foi configurada inicialmente para fornecer às cargas uma tensão de $127 \mathrm{~V}$ com ângulo de fase em $90^{\circ} \mathrm{e}$ freqüência em $60 \mathrm{~Hz}$. Toda a configuração da fonte foi realizada por meio de um microcomputador com o software Cigui32 iX Series (R)(California Instruments, 2007), em que todos os ajustes são transmitidos à fonte por uma interface serial $R S-232$.

\subsection{Analisadores de Energia}

Os analisadores de energia utilizados para extração das assinaturas harmônicas foram dois, onde um deles encontra-se sob o mesmo chassi da fonte de alimentação. Portanto, este equipamento possui também como característica a função de analisador de energia. $\mathrm{O}$ segundo analisador utilizado foi um Fluke 435 (Fluke, 2006).

A utilização de dois analisadores foi adotada a fim de se obter uma maior quantidade de medidas, a qual auxilia no treinamento das redes neurais, além também de se considerar neste processo os desvios marginais das medições de um equipamento em relação ao outro. Adicionalmente, o uso de dois analisadores possibilitou ainda verificar se, para medições realizadas em equipamentos diferentes (mesmo com baixos níveis de distinção), as redes neurais conseguiriam identificar os perfis de cargas conectados no sistema elétrico.

Ambos os analisadores foram gerenciados via software para que a aquisição dos dados fosse realizada de modo semiautomático, sendo que neste caso o operador fica apenas responsável pela disparada dos procedimentos de coleta. No caso do analisador da California Instruments, os dados foram acessados e resgatados via interface serial e então armazenados no microcomputador.

Em relação ao analisador da Fluke, os dados foram obtidos via interface serial opto-isolada, que é convertida para uma interface USB (Universal Serial Bus), realizando-se assim a conexão com o microcomputador, cujos dados são compilados pelo software FlukeView $囚($ Fluke, 2005). 


\subsection{Bancada de Cargas}

Como enfatizado nas seções anteriores, o sistema elétrico sob estudo é considerado residencial, principalmente pela natureza das cargas analisadas.

As características de cada uma das cargas utilizadas nos ensaios laboratoriais são descritas pela Tabela 2.

Tabela 2: Características das cargas empregadas nos ensaios laboratoriais.

\begin{tabular}{|c|c|c|c|}
\hline $\begin{array}{c}\text { Tipo de } \\
\text { Carga }\end{array}$ & $\begin{array}{c}\text { Tensão } \\
\text { (V) }\end{array}$ & $\begin{array}{c}\text { Potência } \\
\text { Nominal(W) }\end{array}$ & $\begin{array}{c}\text { Frequiência } \\
\text { (Hz) }\end{array}$ \\
\hline $\begin{array}{c}\text { Lâmpada In- } \\
\text { candescente }\end{array}$ & 127 & 100 & 60 \\
\hline $\begin{array}{c}\text { Lâmpada } \\
\text { Fluorescente }\end{array}$ & 127 & 20 & 60 \\
\hline $\begin{array}{c}\text { Lâmpada } \\
\text { Fluorescente } \\
\text { Compacta }\end{array}$ & $110 / 127$ & 23 & $50 / 60$ \\
\hline Ventilador & 127 & 120 & 60 \\
\hline $\begin{array}{c}\text { Computador } \\
\text { Pessoal }\end{array}$ & $115 / 230$ & 400 & $50 / 60$ \\
\hline Monitor & $110 / 240$ & 450 & $50 / 60$ \\
\hline
\end{tabular}

As informações apresentadas na Tabela 2 denotam os dados fornecidos pelos fabricantes. Desta forma, por meio da Tabela 3, são mostradas as informações sobre potência ativa, aparente e fator de potência de cada uma das cargas no momento em que estas se encontravam em regime permanente, isto é, sob as condições nas quais foram realizadas as aquisições dos dados.

Com relação às cargas utilizadas, cabe comentar que a lâmpada fluorescente foi acionada por um reator eletrônico com potência nominal de $40 \mathrm{~W}$, o que explica a potência ativa medida na mesma.

\section{SELETORES DE ATRIBUTOS}

As devidas considerações com relação aos seletores de atributos serão apresentadas nesta seção.

Para o desenvolvimento deste trabalho, foram aplicados três seletores de atributos distintos, com o intuito de verificar seus desempenhos.

O processo de seleção de atributos é definido por Liu e Motoda (1998) como a determinação de um subconjunto de atributos que será responsável por generalizar a informação contida na base de dados, isto de acordo com a resposta que se
Tabela 3: Características das cargas quando mantidas em regime permanente e medidas pelos analisadores de energia.

\begin{tabular}{|c|c|c|c|}
\hline $\begin{array}{c}\text { Tipo de } \\
\text { Carga }\end{array}$ & $\begin{array}{c}\text { Potência } \\
\text { Ativa } \\
\text { (W) }\end{array}$ & $\begin{array}{c}\text { Potência } \\
\text { Aparente } \\
\text { (VA) }\end{array}$ & $\begin{array}{c}\text { Fator de } \\
\text { Potência }\end{array}$ \\
\hline $\begin{array}{c}\text { Lâmpada In- } \\
\text { candescente }\end{array}$ & 100 & 100 & 1,000 \\
\hline $\begin{array}{c}\text { Lâmpada } \\
\text { Fluorescente }\end{array}$ & 29 & 50 & 0,580 \\
\hline $\begin{array}{c}\text { Lâmpada } \\
\text { Fluorescente } \\
\text { Compacta }\end{array}$ & 23 & 41 & 0,561 \\
\hline Ventilador & 110 & 169 & 0,651 \\
\hline $\begin{array}{c}\text { Computador } \\
\text { Pessoal }\end{array}$ & 93 & 167 & 0,557 \\
\hline Monitor & 53 & 97 & 0,546 \\
\hline
\end{tabular}

espera obter de tal base de dados.

Seletores de atributos são muito empregados quando os algoritmos de classificação não conseguem obter a generalização de padrões, ou ainda, quando estes algoritmos são inviabilizados devido à grande quantidade de atributos que seriam inseridos como entrada. No estudo em questão, as duas premissas são validas, pois, quando as RNAs são treinadas com todas as correntes harmônicas, estas não conseguem generalizar a solução do problema; além disso, há também uma grande quantidade de atributos que torna o treinamento das RNAs bastante ineficiente.

Geralmente, utiliza-se a seleção de atributos para evitar estas duas hipóteses, pois, ao se reduzir o número de entradas, minimiza-se também o espaço de busca pela melhor solução.

Os métodos para seleção de atributos podem ser divididos em duas classes: wrappers (Kohavi e John, 1997) e filtros (Almuallim e Dietterich, 1991; Liu e Setiono, 1996). Filtros diferenciam-se de wrappers apenas quanto à independência do algoritmo de aprendizado que será utilizado posteriormente. Normalmente, wrappers apresentam desempenho considerável quando o algoritmo de aprendizado é supervisionado (Liu e Setiono, 1996; Hall, 2000). O desempenho dos wrappers foi anteriormente citado como considerável devido aos testes de desempenho realizados no trabalho de Kohavi e John (1997), em que diversas bases de dados comumente empregadas para benchmark foram utilizadas e verificou-se o bom desempenho deste seletor de atributos.

Durante o desenvolvimento deste trabalho foram empregados 
métodos de ambas as classes, com intuito de se comprovar os benefícios fornecidos em cada tipo, além de fornecer uma análise de desempenho dos mesmos.

Alguns autores defendem a idéia de que a seleção de atributos é um ponto central para tarefas de classificação de padrões, visto que muitas vezes a não seleção dos atributos pode levar os classificadores a uma identificação com baixo desempenho e alto custo computacional (Hall e Holmes, 2003). Assim, no que se segue serão apresentados os três métodos para seleção de atributos empregados, os quais foram executados com o auxílio da ferramenta computacional WEKAC) (Waikato Environment Knowledge Analysis) (Witten e Frank, 2005).

\subsection{Wrapper}

Seletores de atributos do tipo Wrapper são muito utilizados quando se deseja selecionar atributos em problemas de aprendizado supervisionado, isto é, quando há uma saída desejada para o sistema. A metodologia consiste na apresentação de um conjunto de treinamento em que os atributos passam pelo método de busca pré-determinado pelo usuário. Após a busca de um subconjunto de atributos, este é avaliado e recebe um custo. Porém, para que o método tenha a devida validade, estes subconjuntos devem ser classificados por um algoritmo de indução também pré-definido pelo usuário, que fornecerá como resultado uma estimativa de desempenho. Após todos os subconjuntos de atributos serem submetidos ao mesmo processo, os melhores subconjuntos são selecionados e deverão novamente passar por um segundo algoritmo de indução que irá extrair os atributos mais relevantes, sendo estes avaliados junto a um conjunto de validação a fim de que os atributos selecionados mostrem seu devido potencial (Kohavi e John, 1997).

Este algoritmo foi empregado devido ao fato de normalmente fornecer melhores resultados do que filtros, isto porque seu método de busca interage com o algoritmo de indução que realiza uma prévia avaliação dos subconjuntos de atributos. Entretanto, como mostrado por Hall e Holmes (2003), métodos do tipo Wrapper apresentam alto custo computacional devido ao algoritmo de indução ser executado para todos os subconjuntos analisados.

Para o trabalho em questão empregou-se uma árvore de decisão do tipo J48 (Goldschmidt e Passos, 2005) tanto como método de busca quanto como algoritmo de indução.

\subsection{Consistency-based Filter (CF)}

O método $C F$, proposto por Liu e Setiono (1996), avalia os subconjuntos de atributos conforme sua consistência em relação às classes que compõem o conjunto de dados e, diferen- temente da grande maioria dos métodos para seleção de atributos, não utiliza busca heurística, mas sim uma busca probabilística baseada no algoritmo Las Vegas (Mitzenmacher e Upfal, 2005). Conforme os resultados experimentais obtidos por Liu e Setiono (1996), pôde-se verificar que este método geralmente obtém uma resposta rápida e garante também a localização de um subconjunto de atributos que forneça boa generalização da informação contida na base de dados, além de ser facilmente implementado.

O algoritmo Las Vegas faz escolhas probabilísticas que auxiliam no processo de busca; assim, encontra-se rapidamente um subconjunto de atributos que forneça resultados satisfatórios. Esta busca é executada até que um número máximo de tentativas seja alcançado, sendo que ao final dessas tentativas avaliam-se tanto o tamanho do subconjunto de atributos como a sua inconsistência com relação à classe. O subconjunto selecionado será o de menor tamanho e inconsistência, visto que a consistência de um subconjunto de atributos é inversamente proporcional à sua inconsistência.

Uma desvantagem apresentada pela busca probabilística quando comparada a uma busca heurística é o seu custo computacional um pouco mais alto. No entanto, sua maior vantagem é que não possui a mesma vulnerabilidade apresentada pela busca heurística quando submetido a conjuntos de dados com muitos atributos correlatos.

\subsection{Correlation-based Feature Selection (CFS)}

O CFS é um método proposto por Hall (1999), que pode ser aplicado tanto em conjuntos de dados contínuos como discretos. O método faz uso de correlação para avaliar o custo dos atributos. Contudo, uma grande diferença apresentada pelo $C F S$, quando comparado a outros filtros, é que sua seleção começa pela avaliação dos subconjuntos de atributos para depois avaliar os atributos individualmente.

A busca pelo melhor subconjunto de atributos é finalizada apenas quando o critério de parada é satisfeito, sendo este factível quando as últimas cinco iterações retornam o mesmo subconjunto de atributos.

Algumas vantagens apresentadas pelo algoritmo $C F S$ são a sua rápida execução, a possibilidade de ser aplicado em qualquer tipo de conjunto de atributos e a redução de até $50 \%$ da quantidade de atributos (Hall, 2000).

\subsection{Análise de Desempenho dos Seleto- res de Atributos}

Antes de apresentar devidamente os resultados obtidos pelos seletores de atributos, cabe-se comentar que todos fo- 
ram executados pela ferramenta computacional WEKA(C), utilizando-se de um microcomputador com 3 GB de memória e com processador Intel $\left(\right.$ Core $^{\mathrm{TM}} 2$ Duo de $1,83 \mathrm{GHz}$. As medidas de desempenho desses métodos podem ser avaliadas por meio da Tabela 4.

Tabela 4: Desempenho dos seletores de atributos.

\begin{tabular}{|c|c|c|}
\hline $\begin{array}{c}\text { Seletores de } \\
\text { Atributos }\end{array}$ & Ranking de Atributos & Tempo (s) \\
\hline$C F S$ & $\begin{array}{c}01,03,08,09,05,07,19, \\
11,17,13,23,25,06,15, \\
10,12,20,22,24,04,21, \\
18,14,02,16\end{array}$ & 0,47 \\
\hline$C F$ & $\begin{array}{c}01,03,08,09,05,07,19, \\
11,17,13,23,25,06,15, \\
10,12,20,22,24,04,21, \\
18,14,02,16\end{array}$ & 0,82 \\
\hline Wrapper & $\begin{array}{c}01,03,08,09,05,07,17 \\
11,13,15,19,20,22,25 \\
18,16,24,14,23,04,10 \\
12,06,21,02\end{array}$ & $\begin{array}{l}175209( \\
\approx 2 \text { dias })\end{array}$ \\
\hline
\end{tabular}

As três técnicas utilizadas foram configuradas de modo a fornecerem como resposta uma lista dos atributos, ordenada do atributo de maior para o de menor relevância. Desta forma, pela lista apresentada na Tabela 4, pôde-se verificar que os seis primeiros atributos mais relevantes foram os mesmos para as três técnicas. Assim, apenas estes seis atributos foram selecionados para compor a nova base de dados.

Outra característica observada é o esforço computacional apresentado por cada um dos algoritmos. O método Wrapper gastou cerca de 2 dias para obter os atributos mais relevantes, enquanto que os algoritmos $C F S$ e $C F$, os quais são baseados em filtros, apresentaram respostas favoráveis num tempo menor do que 1 segundo. Também se pode verificar que o método $C F$ apresenta um tempo maior de resposta do que o método $C F S$. Isto, devido aos seus distintos algoritmos de busca (probabilística e heurística, respectivamente). No entanto, conforme comentado na Seção 2, o tempo despendido pelos seletores de atributos não é relevante devido ao fato de serem somente empregados na etapa de projeto da metodologia proposta.

Com a aplicação das técnicas de seleção de atributos, o espaço de busca pôde ser então efetivamente reduzido. Assim, pela Figura 13, ilustra-se o espaço de busca contendo 60 instâncias e os 25 atributos da base original, sendo que pela Figura 14 pode-se visualizar o espaço de busca contendo as mesmas 60 instâncias, porém, contendo apenas os atributos selecionados.

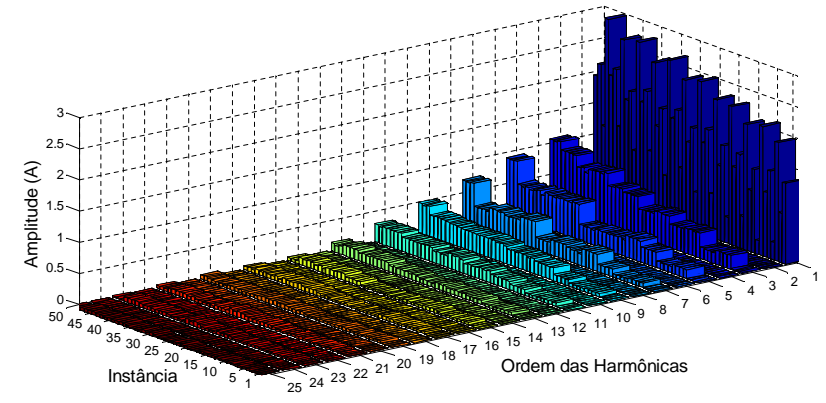

Figura 13: Espaço de busca antes de selecionar os atributos mais relevantes.

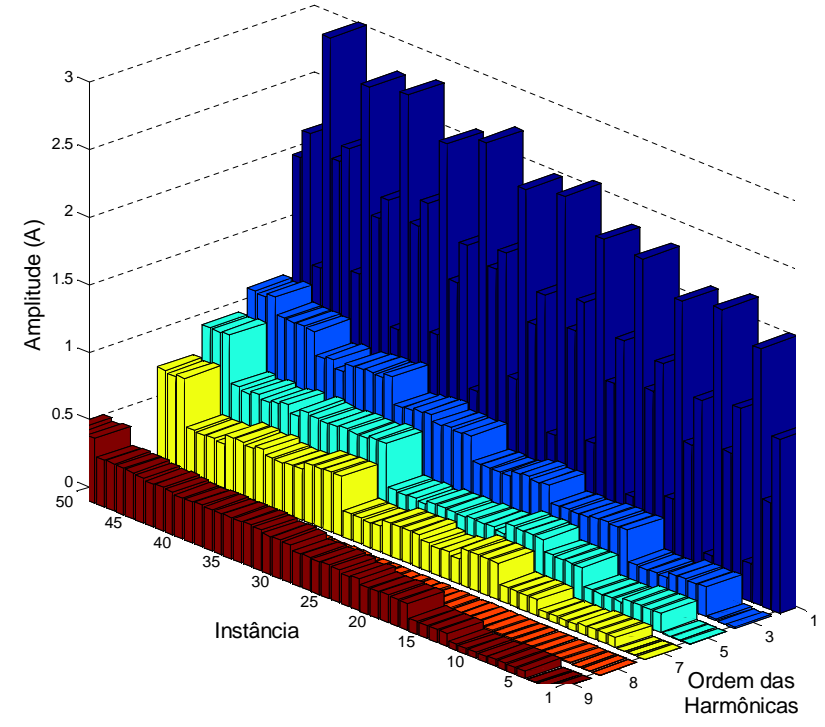

Figura 14: Espaço de busca contendo apenas os atributos selecionados.

O próximo passo considerado, após a seleção dos atributos, foi o treinamento das RNAs, conforme apresentado na seqüência.

\section{REDES NEURAIS ARTIFICIAIS}

As RNAs possuem como principais características a capacidade de aprender por meio de exemplos e a de generalizar as informações aprendidas. A generalização está atrelada com a capacidade de a rede aprender pela apresentação de um conjunto reduzido de exemplos, em que a mesma deverá estar apta a fornecer respostas coerentes para dados que não tenham sido utilizados em sua etapa de aprendizado. Outras características como tolerância a falhas, auto-organização e capacidade de agrupar ou organizar dados também podem ser observadas em algumas arquiteturas de redes neurais (Haykin, 1999). 


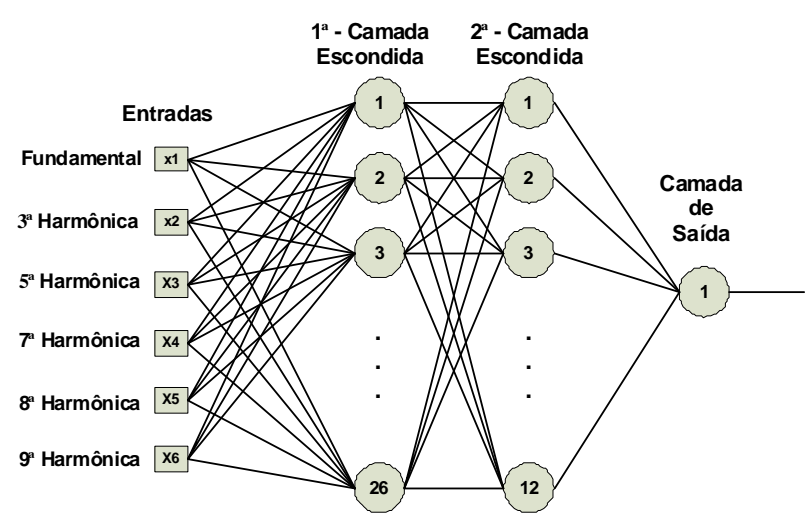

Figura 15: Arquitetura das RNAs.

As RNAs empregadas neste trabalho possuem arquitetura $M L P$, a qual normalmente é aplicada em tarefas de reconhecimento de padrões, aproximação funcional, identificação e controle. Assim, considerando o propósito do reconhecimento de padrões referente à composição harmônica das cargas e suas combinações, verifica-se que tal arquitetura se encaixa à tarefa proposta.

Na intenção de melhorar o processo de identificação, foi criada uma RNA para cada carga utilizada. Vale comentar que todas as RNAs possuem a mesma configuração, sendo 26 neurônios na primeira camada escondida, 12 neurônios na segunda camada escondida e apenas um neurônio na camada de saída. Quanto às funções de ativação, para as camadas escondidas utilizou-se a tangente hiperbólica, sendo que para a camada de saída empregou-se a função linear com limites em 1 e -1 , representando assim o estado ao qual se encontra o equipamento (ligado ou desligado). As funções de ativação têm como finalidade limitar a saída dos neurônios. Ressalta-se ainda que cada uma das RNAs possui 6 entradas que correspondem às amplitudes das correntes fundamental e harmônicas de $3^{a}, 5^{a}, 7^{a}, 8^{a}$ e $9^{a}$ ordens, as quais foram previamente fornecidas como resposta pelos seletores de atributos. Para uma melhor compreensão da arquitetura supracitada, a mesma encontra-se ilustrada por meio da Figura 15.

Com relação ao treinamento das RNAs, muitos algoritmos podem ser encontrados na literatura. Dentre alguns, o mais conhecido é o backpropagation que possui como fundamento o cálculo do gradiente descendente. No entanto, em Hagan e Menhaj (1994), um algoritmo denominado LevenbergMarquardt, que consiste em uma aproximação do método de Newton, se destaca por apresentar um processo de ajuste otimizado dos pesos e uma maior velocidade de convergência quando comparado com algoritmos convencionais como o backpropagation. Esta melhoria na velocidade de convergência apresentada pelo algoritmo de treinamento de LevenbergMarquardt é devida à exploração de informações relaciona- das às derivadas de segunda ordem do erro quadrático, ao passo que o algoritmo de treinamento backpropagation faz apenas uso de informações associadas às derivadas de primeira ordem.

Comenta-se que todas as RNAs empregadas neste estudo, bem como seus algoritmos de treinamento, foram configuradas utilizando-se o Toolbox da ferramenta computacional Matlab ${ }^{\circledR}$ (Demuth et al., 2007).

Apresentadas as arquiteturas das RNAs utilizadas e suas características, na próxima seção serão apontados e discutidos os resultados decorrentes desta aplicação.

\section{RESULTADOS EXPERIMENTAIS}

Nesta seção serão abordados os resultados apresentados pelas RNAs quando submetidas a dois modos de treinamentos e validações, onde o conjunto de dados foi dividido de forma distinta.

\subsection{Primeiro Modo de Treinamento e Vali- dação}

Para o primeiro modo, empregou-se um conjunto de treinamento composto pelas seguintes medições:

- medições com as cargas alimentadas pelas formas de onda contendo distorções harmônicas acima dos limites recomendados pelo IEEE (189 amostras);

- medições realizadas quando as cargas foram alimentadas por tensão sem distorção harmônica (63 amostras);

- medições obtidas pelo analisador da California Instruments quando houve variação na tensão de alimentação entre $\pm 15 \%$ (252 amostras);

- metade das medições adquiridas pelo analisador da Fluke quando as cargas foram alimentadas com tensões distorcidas dentro dos limites recomendados pelo IEEE (63 amostras);

- além de aproximadamente $67 \%$ das medidas adquiridas pelo analisador da California Instruments quando as cargas foram alimentadas com tensões distorcidas dentro dos limites comentados (378 amostras).

Desta forma, o conjunto de treinamento foi composto por um total de 945 amostras, que correspondem em cerca de $68 \%$ da base de dados.

Os dados restantes foram utilizados para compor o conjunto de validação. Cabe-se evidenciar que as medidas obtidas 
quando as cargas foram alimentadas pela forma de onda da Figura 2 fizeram parte do conjunto de validação. Isto porque a mesma apresenta um achatamento na tensão, característico de componentes harmônicas de $3^{a}$ e $5^{a}$ ordens, que não se encontra pronunciado nas demais formas de onda. Essas medidas também foram consideradas devido a esta forma de onda extrapolar os limites de distorção recomendados pelo IEEE.

Sendo assim, após o treinamento das RNAs, estas foram submetidas à etapa de validação, onde foram obtidos os resultados apresentados por meio da Tabela 5 .

Tabela 5: Resultados obtidos com o primeiro modo de treinamento e validação.

\begin{tabular}{|c|c|}
\hline Carga & Acertos (\%) \\
\hline Lâmpada Incandescente & 99,77 \\
\hline Lâmpada Fluorescente & 96,15 \\
\hline Lâmpada Fluorescente Compacta & 96,15 \\
\hline Ventilador & 99,77 \\
\hline Computador Pessoal & 100 \\
\hline Monitor & 99,55 \\
\hline
\end{tabular}

A partir da análise da Tabela 5, nota-se que a RNA responsável por identificar o computador pessoal obteve um aproveitamento de $100 \%$. Ou seja, conseguiu identificar corretamente se a carga estava conectada ou não ao sistema elétrico para todas as 441 instâncias que comportaram o conjunto de validação do primeiro modo de treinamento. Já no caso do ventilador, monitor e lâmpada incandescente, suas RNAs apresentaram resultados acima de $99 \%$, isto é, o erro destas RNAs foi bastante baixo. Apenas as RNAs responsáveis pelas lâmpadas, fluorescente compacta e fluorescente acionada por reator eletrônico, apresentaram taxas de acerto acima de $96 \%$, sendo esta taxa considerada satisfatória. A seguir, por meio da Tabela 6, são mostradas as instâncias onde as cargas foram classificadas incorretamente.

Para a análise da tabela 6 , a mesma nomenclatura utilizada na Tabela 1 foi adotada; entretanto, neste caso, esta nomenclatura refere-se às RNAs responsáveis por cada uma das cargas. Os " $\mathrm{X}$ " denotam cargas ligadas e "O" cargas desligadas. Esta tabela representa a saída desejada pelas RNAs. Contudo, para uma melhor compreensão dos erros cometidos pelas RNAs, os "X" e "O" em destaque representam as situações nas quais as RNAs apresentaram respostas errôneas, ou seja, a saída da rede neural foi contrária à saída apresentada na tabela. Por exemplo, na instância 91, as cargas conectadas ao sistema eram: MON, LFC e LF; no entanto, a saída da RNA responsável pela LFC apresentou uma resposta onde tal lâmpada encontrava-se desligada.
Tabela 6: Instâncias do conjunto de validação 1 identificadas de forma errônea.

\begin{tabular}{|c|c|c|c|c|c|c|}
\hline \multirow{2}{*}{ Instâncias } & \multicolumn{6}{|c|}{ Conjunto de Validação 1} \\
\hline & PC & MON & LFC & $\mathbf{L F}$ & LI & VE \\
\hline 91 & $\mathrm{O}$ & $\mathrm{X}$ & $\mathbf{X}$ & $\mathrm{X}$ & $\mathrm{O}$ & $\mathrm{O}$ \\
\hline 165 & $X$ & $\mathrm{O}$ & $\mathbf{O}$ & $\mathrm{X}$ & $X$ & $\mathrm{X}$ \\
\hline 179 & $\mathrm{X}$ & $X$ & $\mathbf{O}$ & $\mathbf{X}$ & $\mathrm{O}$ & $\mathrm{X}$ \\
\hline 180 & $\mathrm{X}$ & $\mathrm{X}$ & $\mathbf{O}$ & $\mathrm{X}$ & $\mathrm{X}$ & $\mathrm{O}$ \\
\hline 231 & $\mathrm{X}$ & $\mathrm{O}$ & $\mathbf{X}$ & $\mathrm{O}$ & $\mathrm{X}$ & $\mathrm{O}$ \\
\hline 236 & $\mathrm{X}$ & $\mathrm{O}$ & $\mathrm{X}$ & $\mathbf{X}$ & $\mathrm{X}$ & $\mathrm{X}$ \\
\hline 240 & $\mathrm{X}$ & $\mathrm{X}$ & $\mathrm{O}$ & $\mathbf{O}$ & $\mathrm{X}$ & $\mathrm{X}$ \\
\hline 246 & $\mathrm{X}$ & $\mathrm{X}$ & $\mathrm{X}$ & $\mathbf{O}$ & $\mathrm{O}$ & $\mathrm{X}$ \\
\hline 247 & $\mathrm{X}$ & $\mathrm{X}$ & $\mathrm{X}$ & $\mathbf{O}$ & $\mathrm{X}$ & $\mathrm{O}$ \\
\hline 314 & $\mathrm{X}$ & $\mathrm{X}$ & $\mathrm{X}$ & $X$ & $\mathbf{X}$ & $\mathrm{O}$ \\
\hline 385 & $\mathrm{O}$ & $\mathrm{O}$ & $\mathrm{O}$ & $\mathbf{X}$ & $X$ & $X$ \\
\hline 387 & $\mathrm{O}$ & $\mathrm{O}$ & $\mathbf{X}$ & $\mathrm{O}$ & $\mathrm{O}$ & $\mathrm{X}$ \\
\hline 390 & $\mathrm{O}$ & $\mathrm{O}$ & $\mathbf{X}$ & $X$ & $\mathrm{O}$ & $\mathrm{O}$ \\
\hline 393 & $\mathrm{O}$ & $\mathbf{O}$ & $X$ & $\mathbf{X}$ & $X$ & $\mathrm{X}$ \\
\hline 396 & $\mathrm{O}$ & $X$ & $\mathbf{O}$ & $\mathrm{O}$ & $X$ & $\mathrm{O}$ \\
\hline 398 & $\mathrm{O}$ & $X$ & $\mathbf{O}$ & $X$ & $\mathrm{O}$ & $\mathrm{O}$ \\
\hline 399 & $\mathrm{O}$ & $X$ & $\mathrm{O}$ & $\mathbf{X}$ & $\mathrm{O}$ & $\mathrm{X}$ \\
\hline 401 & $\mathrm{O}$ & $X$ & $\mathrm{O}$ & $\mathbf{X}$ & $X$ & $X$ \\
\hline 403 & $\mathrm{O}$ & $X$ & $\mathbf{X}$ & $\mathrm{O}$ & $\mathrm{O}$ & $X$ \\
\hline 404 & $\mathrm{O}$ & $\mathbf{X}$ & $X$ & $\mathbf{O}$ & $X$ & $\mathrm{O}$ \\
\hline 413 & $X$ & $\mathrm{O}$ & $\mathbf{O}$ & $\mathrm{O}$ & $X$ & $X$ \\
\hline 415 & $X$ & $\mathrm{O}$ & O & $X$ & $\mathrm{O}$ & $X$ \\
\hline 416 & $X$ & $\mathrm{O}$ & O & $X$ & $X$ & $\mathrm{O}$ \\
\hline 417 & $X$ & $\mathrm{O}$ & $\mathrm{O}$ & $\mathbf{X}$ & $X$ & $X$ \\
\hline 418 & $X$ & $\mathrm{O}$ & $\mathbf{X}$ & O & $\mathrm{O}$ & $\mathrm{O}$ \\
\hline 419 & $X$ & $\mathrm{O}$ & $X$ & $\mathbf{O}$ & $\mathrm{O}$ & $X$ \\
\hline 420 & $X$ & $\mathrm{O}$ & $X$ & $\mathbf{O}$ & $X$ & $\mathrm{O}$ \\
\hline 426 & $X$ & $X$ & $\mathbf{O}$ & $\mathrm{O}$ & $\mathrm{O}$ & $\mathrm{O}$ \\
\hline 429 & $X$ & $X$ & $\mathrm{O}$ & $\mathbf{O}$ & $X$ & $X$ \\
\hline 434 & $X$ & $X$ & $\mathbf{X}$ & $\mathbf{O}$ & $\mathrm{O}$ & $\mathrm{O}$ \\
\hline 435 & $X$ & $X$ & $\mathbf{X}$ & $\mathrm{O}$ & $\mathrm{O}$ & $X$ \\
\hline 437 & $X$ & $X$ & $X$ & $\mathbf{O}$ & $X$ & $X$ \\
\hline 439 & $X$ & $X$ & $X$ & $X$ & $\mathrm{O}$ & $\mathbf{X}$ \\
\hline
\end{tabular}

Ainda com relação à Tabela 6 , cabe comentar que apenas um erro foi gerado quando da apresentação de medidas realizadas com desvios na alimentação das cargas entre $\pm 15 \%$ da 
tensão (instância 91). Os demais erros foram todos ocasionados quando se alimentou as cargas com formas de onda dis-

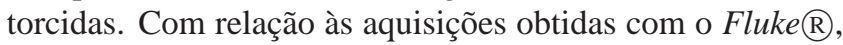
estas também foram submetidas às redes neurais, porém, não apresentaram erros. Nota-se ainda que as instâncias 240 e 429 possuem a mesma saída desejada e apresentaram o mesmo erro; porém, a primeira instância foi adquirida quando as cargas foram alimentadas por uma forma de onda com DHT de 3,02\%, ao passo que a segunda instância foi medida quando as cargas foram alimentadas por uma forma de onda com DHT de 8,12\% (Figura 5). Cabe também comentar que apenas nas instâncias 179, 393, 404, 418 e 434 os erros foram cometidos por duas redes neurais distintas.

\subsection{Segundo Modo de Treinamento e Va- lidação}

Para o segundo modo, o conjunto de treinamento foi alterado apenas com relação à quantidade de medidas adquiridas pelo analisador da California Instruments, quando as cargas foram alimentadas com tensões distorcidas dentro dos limites recomendados pelo IEEE. Utilizou-se para tanto de aproximadamente $44 \%$ destas medidas, isto é, apenas 252 amostras ao invés de 378 amostras, conforme realizado no primeiro modo de treinamento e validação. Assim, o conjunto de treinamento foi composto por um total de 819 amostras, que corresponde a cerca de $59 \%$ da base de dados. Os dados restantes foram utilizados durante a etapa de validação das RNAs.

Apresentam-se por meio da Tabela 7 os resultados obtidos após a validação das RNAs para o segundo modo de treinamento.

Tabela 7: Resultados obtidos com o segundo modo de treinamento e validação.

\begin{tabular}{|c|c|}
\hline Carga & Acertos (\%) \\
\hline Lâmpada Incandescente & 99,65 \\
\hline Lâmpada Fluorescente & 95,59 \\
\hline Lâmpada Fluorescente Compacta & 94,18 \\
\hline Ventilador & 99,65 \\
\hline Computador Pessoal & 100 \\
\hline Monitor & 99,12 \\
\hline
\end{tabular}

Quanto ao segundo modo de treinamento e validação, pôdese observar por meio da Tabela 7 que houve um decréscimo de qualidade nos resultados quando comparados àqueles obtidos no primeiro modo de treinamento e validação (Tabela 5). No entanto, verifica-se que esta metodologia, apesar de apresentar resultados menos favoráveis, ainda pode ser con- siderada satisfatória para a identificação de cargas lineares e não-lineares. As instâncias classificadas incorretamente por cada uma das RNAs são ilustradas por meio da Tabela 8.

Para análise desta tabela, foram utilizadas as mesmas nomenclaturas e representações da Tabela 6; no entanto, esta reflete o segundo modo de treinamento e validação.

Ao se examinar a Tabela 8, verificou-se que não houve erros quando da classificação das medidas obtidas com o Fluke $\mathbb{R}$ e também para aquelas adquiridas quando as cargas foram alimentadas com desvio de tensão entre $\pm 15 \%$. Portanto, os erros encontrados referem-se às medições realizadas com o California Instruments quando as cargas foram alimentadas por tensões distorcidas, tanto aquelas que se encontram dentro dos limites de distorção estipulados pelo IEEE quanto pelas formas de onda que extrapolam estes limites. Em alguns casos, nota-se que duas redes neurais distintas apresentaram respostas errôneas em uma mesma instância (instâncias 179, 242, 518, 519, 530, 541, 542, 543, 544, 560 e 562), onde se verificou que estes erros foram geralmente apresentados pelas RNAs responsáveis por classificar as lâmpadas fluorescente e fluorescente compacta. Também pôde ser verificado que apenas na instância 517, três RNAs apresentaram respostas errôneas. Em algumas instâncias verificou-se que a resposta desejada pelas RNAs era igual, no entanto, as cargas foram alimentadas com formas de onda distintas. As instâncias 147 e 525 ; 148 e 526 são casos em que as cargas foram alimentadas com tensões distorcidas distintas, as quais possuíam DHT de 4,42\% e 8,12\% (Figura 5), respectivamente. Nas instâncias 269 e 521; 303 e 555 ocorrem o mesmo, entretanto, as cargas são alimentadas com formas de onda contendo DHTs respectivamente de 3,02\% e 8,12\% (Figura 5). Já as instâncias 146 e 209; 179 e 242 também são iguais, porém, as DHTs das formas de onda foram respectivamente de $4,42 \%$ e 3,94\%. Além disso, as instâncias 162 e 351 foram alimentadas por formas de onda distorcidas distintas, as quais apresentavam DHTs de tensão de respectivamente $4,42 \%$ e $3,02 \%$.

\section{CONCLUSÕES}

Neste trabalho apresentou-se um estudo sobre a identificação de cargas lineares e não-lineares em sistemas elétricos residenciais, em que foi gerada uma base de dados a partir de ensaios laboratoriais. Os ensaios forneceram dados reais de cargas comumente utilizadas em residências, os quais possibilitaram o treinamento e validação das RNAs. Estes dados foram adquiridos a partir de formas de onda distorcidas e também de formas de onda com variações de tensão entre $\pm 15 \%$. Tais ensaios tornaram viável a aplicação de redes neurais, pois, se fossem utilizados apenas dados representando a condição normal do sistema, não seria então possível realizar o treinamento e validação das mesmas devido ao pe- 
Tabela 8: Instâncias do conjunto de validação 2 identificadas de forma errônea.

\begin{tabular}{|c|c|c|c|c|c|c|}
\hline \multirow{2}{*}{ Instâncias } & \multicolumn{6}{|c|}{ Conjunto de Validação 2} \\
\hline & $\mathbf{P C}$ & MON & LFC & $\mathbf{L F}$ & LI & VE \\
\hline 130 & $\mathrm{O}$ & $\mathrm{O}$ & $\mathbf{O}$ & $\mathrm{X}$ & $\mathrm{O}$ & $\mathrm{O}$ \\
\hline 138 & $\mathrm{O}$ & $\mathrm{O}$ & $\mathbf{X}$ & $\mathrm{X}$ & $\mathrm{O}$ & $\mathrm{O}$ \\
\hline 146 & $\mathrm{O}$ & $\mathrm{X}$ & $\mathbf{O}$ & $\mathrm{X}$ & $\mathrm{O}$ & $\mathrm{O}$ \\
\hline 147 & $\mathrm{O}$ & $\mathrm{X}$ & $\mathbf{O}$ & $\mathrm{X}$ & $\mathrm{O}$ & $\mathrm{X}$ \\
\hline 148 & $\mathrm{O}$ & $\mathrm{X}$ & $\mathbf{O}$ & $X$ & $\mathrm{X}$ & $\mathrm{O}$ \\
\hline 162 & $\mathrm{X}$ & $\mathrm{O}$ & $\mathbf{O}$ & $\mathrm{X}$ & $\mathrm{O}$ & $\mathrm{O}$ \\
\hline 164 & $\mathrm{X}$ & $\mathrm{O}$ & $\mathbf{O}$ & $\mathrm{X}$ & $\mathrm{X}$ & $\mathrm{O}$ \\
\hline 165 & $\mathrm{X}$ & $\mathrm{O}$ & $\mathbf{O}$ & $\mathrm{X}$ & $\mathrm{X}$ & $\mathrm{X}$ \\
\hline 179 & $\mathrm{X}$ & $\mathrm{X}$ & $\mathbf{O}$ & $\mathbf{X}$ & $\mathrm{O}$ & $\mathrm{X}$ \\
\hline 181 & $\mathrm{X}$ & $X$ & $\mathbf{O}$ & $X$ & $X$ & $\mathrm{X}$ \\
\hline 188 & $\mathrm{X}$ & $X$ & $\mathrm{X}$ & $X$ & $\mathbf{X}$ & $\mathrm{O}$ \\
\hline 209 & $\mathrm{O}$ & $X$ & $\mathbf{O}$ & $X$ & $\mathrm{O}$ & $\mathrm{O}$ \\
\hline 241 & $X$ & $X$ & O & $X$ & $\mathrm{O}$ & $\mathrm{O}$ \\
\hline 242 & $X$ & $X$ & O & $\mathbf{X}$ & $\mathrm{O}$ & $X$ \\
\hline 243 & $X$ & $X$ & $\mathrm{O}$ & $\mathbf{X}$ & $X$ & $\mathrm{O}$ \\
\hline 251 & $X$ & $X$ & $X$ & $X$ & $X$ & O \\
\hline 269 & $\mathrm{O}$ & $X$ & $\mathrm{O}$ & O & $\mathrm{O}$ & $X$ \\
\hline 287 & $X$ & $\mathrm{O}$ & $\mathrm{O}$ & O & $X$ & $X$ \\
\hline 294 & $X$ & $\mathrm{O}$ & $\mathbf{X}$ & $\mathrm{O}$ & $X$ & $\mathrm{O}$ \\
\hline 303 & $X$ & $X$ & $\mathrm{O}$ & O & $X$ & $X$ \\
\hline 309 & $X$ & $X$ & X & O & $\mathrm{O}$ & $X$ \\
\hline 351 & $X$ & $\mathrm{O}$ & O & $X$ & $\mathrm{O}$ & $\mathrm{O}$ \\
\hline 352 & $X$ & $\mathrm{O}$ & O & $X$ & $\mathrm{O}$ & $X$ \\
\hline 431 & $X$ & $X$ & $\mathrm{O}$ & $\mathbf{X}$ & $\mathrm{O}$ & $X$ \\
\hline 509 & $\mathrm{O}$ & $\mathrm{O}$ & O & $X$ & $\mathrm{O}$ & $X$ \\
\hline 511 & $\mathrm{O}$ & $\mathrm{O}$ & O & $X$ & $X$ & $X$ \\
\hline 513 & $\mathrm{O}$ & $\mathrm{O}$ & $\mathbf{X}$ & $\mathrm{O}$ & $\mathrm{O}$ & $X$ \\
\hline 516 & $\mathrm{O}$ & $\mathbf{O}$ & $X$ & $\mathrm{X}$ & $\mathrm{O}$ & $\mathrm{O}$ \\
\hline 517 & $\mathrm{O}$ & O & $\mathbf{X}$ & $\mathbf{X}$ & $\mathrm{O}$ & $X$ \\
\hline 518 & $\mathrm{O}$ & $\mathbf{O}$ & $X$ & $\mathbf{X}$ & $X$ & $\mathrm{O}$ \\
\hline 519 & $\mathrm{O}$ & $\mathbf{O}$ & $X$ & $\mathbf{X}$ & $X$ & $X$ \\
\hline 521 & $\mathrm{O}$ & $X$ & $\mathrm{O}$ & $\mathbf{O}$ & $\mathrm{O}$ & $X$ \\
\hline 523 & $\mathrm{O}$ & $X$ & $\mathbf{O}$ & $\mathrm{O}$ & $X$ & $X$ \\
\hline 525 & $\mathrm{O}$ & $X$ & O & $X$ & $\mathrm{O}$ & $X$ \\
\hline 526 & $\mathrm{O}$ & $X$ & $\mathbf{O}$ & $X$ & $X$ & $\mathrm{O}$ \\
\hline 527 & $\mathrm{O}$ & $X$ & $\mathbf{O}$ & $X$ & $X$ & $X$ \\
\hline 528 & $\mathrm{O}$ & $X$ & $X$ & O & $\mathrm{O}$ & $\mathrm{O}$ \\
\hline 529 & $\mathrm{O}$ & $X$ & $X$ & $\mathbf{O}$ & $\mathrm{O}$ & $X$ \\
\hline
\end{tabular}

\begin{tabular}{|c|c|c|c|c|c|c|}
\hline $\mathbf{5 3 0}$ & $\mathrm{O}$ & $\mathbf{X}$ & $\mathrm{X}$ & $\mathbf{O}$ & $\mathrm{X}$ & $\mathrm{O}$ \\
\hline $\mathbf{5 3 1}$ & $\mathrm{O}$ & $\mathrm{X}$ & $\mathrm{X}$ & O & $\mathrm{X}$ & $\mathrm{X}$ \\
\hline $\mathbf{5 3 7}$ & $\mathrm{X}$ & $\mathrm{O}$ & $\mathbf{O}$ & $\mathrm{O}$ & $\mathrm{O}$ & $\mathrm{X}$ \\
\hline $\mathbf{5 3 9}$ & $\mathrm{X}$ & $\mathrm{O}$ & O & $\mathrm{O}$ & $\mathrm{X}$ & $\mathrm{X}$ \\
\hline $\mathbf{5 4 1}$ & $\mathrm{X}$ & $\mathrm{O}$ & $\mathbf{O}$ & $\mathbf{X}$ & $\mathrm{O}$ & $\mathrm{X}$ \\
\hline $\mathbf{5 4 2}$ & $\mathrm{X}$ & $\mathrm{O}$ & $\mathbf{O}$ & $\mathbf{X}$ & $\mathrm{X}$ & $\mathrm{O}$ \\
\hline $\mathbf{5 4 3}$ & $\mathrm{X}$ & $\mathrm{O}$ & $\mathbf{O}$ & $\mathbf{X}$ & $\mathrm{X}$ & $\mathrm{X}$ \\
\hline $\mathbf{5 4 4}$ & $\mathrm{X}$ & $\mathrm{O}$ & $\mathbf{X}$ & O & $\mathrm{O}$ & $\mathrm{O}$ \\
\hline $\mathbf{5 5 1}$ & $\mathrm{X}$ & $\mathrm{O}$ & $\mathrm{X}$ & $\mathrm{X}$ & $\mathrm{X}$ & $\mathbf{X}$ \\
\hline $\mathbf{5 5 2}$ & $\mathrm{X}$ & $\mathrm{X}$ & $\mathrm{O}$ & O & $\mathrm{O}$ & $\mathrm{O}$ \\
\hline $\mathbf{5 5 5}$ & $\mathrm{X}$ & $\mathrm{X}$ & $\mathrm{O}$ & O & $\mathrm{X}$ & $\mathrm{X}$ \\
\hline $\mathbf{5 6 0}$ & $\mathrm{X}$ & $\mathrm{X}$ & $\mathbf{X}$ & O & $\mathrm{O}$ & $\mathrm{O}$ \\
\hline $\mathbf{5 6 2}$ & $\mathrm{X}$ & $\mathrm{X}$ & $\mathbf{X}$ & O & $\mathrm{X}$ & $\mathrm{O}$ \\
\hline $\mathbf{5 6 3}$ & $\mathrm{X}$ & $\mathrm{X}$ & $\mathrm{X}$ & O & $\mathrm{X}$ & $\mathrm{X}$ \\
\hline $\mathbf{5 6 5}$ & $\mathrm{X}$ & $\mathrm{X}$ & $\mathrm{X}$ & $\mathrm{X}$ & O & $\mathrm{X}$ \\
\hline $\mathbf{5 6 6}$ & $\mathrm{X}$ & $\mathrm{X}$ & $\mathbf{X}$ & $\mathrm{X}$ & $\mathrm{X}$ & $\mathrm{O}$ \\
\hline
\end{tabular}

queno conjunto de dados que seria formado.

Pela impossibilidade de saber quais harmônicas seriam mais relevantes à identificação das cargas, foram então registradas as componentes harmônicas da $1^{a}$ à $25^{a}$ ordens. Desta maneira, a grande quantidade de sinais obtidos para cada assinatura harmônica das cargas e suas combinações fez com que o conjunto de dados tivesse muitos atributos, o que tornou o espaço de busca mais complexo. Assim, técnicas para a seleção de atributos foram aplicadas para melhor preparar os dados que seriam fornecidos como entradas às RNAs, com o intuito de se extrair uma resposta eficiente que determinasse o comportamento dinâmico do sistema.

Os resultados experimentais mostraram que as RNAs apresentam desempenho satisfatório até mesmo sobre condições que extrapolam a normalidade do sistema elétrico, devido à sua capacidade de generalizar o conhecimento. Tais resultados mostram o grande potencial desta técnica, podendo ser muito bem empregadas a problemas de identificação de cargas lineares e não-lineares.

Como trabalho futuro, vislumbra-se a identificação das cargas em tempo-real, por meio de um processador de baixo custo, que seria empregado apenas na extração do espectro da corrente e no envio deste para um computador remoto, o qual seria então responsável por aplicar a metodologia proposta neste trabalho. Cabe-se comentar também que o computador remoto seria responsável por gerenciar um grande conjunto de consumidores. A utilização de tal processador possibilitaria uma expressiva redução de custos, devido à possibilidade 
de se eliminar do sistema de identificação os analisadores de energia, além de tornar o sistema totalmente automatizado. Além disso, o surgimento dos smart meters (medidor inteligente) auxilia significativamente nesta tarefa, visto que o próprio smart meter poderá transmitir tais dados para as concessionárias via rede PLC (Power Line Communication). Ainda como sugestão de trabalho futuro, após o processo de identificação das cargas em tempo-real, tem-se as pesquisas que podem ser direcionadas a fim de quantificar o número de cada um desses equipamentos presentes nas residências.

Por fim, pode-se aplicar este sistema baseado em redes neurais artificiais para a identificação de consumidores que estejam contribuindo para distorções harmônicas na tensão e/ou corrente dos sistemas de distribuição de energia, sendo este o tipo de aplicação comumente encontrado na literatura.

\section{AGRADECIMENTOS}

Os autores gostariam de agradecer à FAPESP, ao CNPq e à CAPES pelos auxílios financeiros propiciados no decorrer das atividades de pesquisas. Agradecimentos especiais também aos revisores frente às valiosas contribuições propiciadas no decorrer do processo de revisão.

\section{REFERÊNCIAS}

Acarkan, B. e Erkan, K. (2007). Harmonics Modeling and Harmonic Activity Analysis of Equipments with Switch Mode Power Supply Using Matlab and Simulink. Electric Machines \& Drives Conference (IEMDC), pp. 508-513.

Almuallim, H. e Dietterich, T. G. (1991). Learning with many Irrelevant Features. Proc. of the $9^{\text {th }}$ National Conference on Artificial Intelligence.

Bezerra, U. H., Tostes, M. E. L., Araújo, A. C. S., Garcez, J. N., Mesquita, J. E. e Tupiassú, A. A. (2001). Harmônicos Gerados por Consumidores em Baixa Tensão da Rede Metropolitana de Distribuição de Energia de Belém (PA). I Congresso de Inovação Tecnológica em Energia Elétrica (Citenel).

California Instruments (2007). AC Power Source - User Manual.

Chan, W. L., So, A. T. P. e Lai, L. L. (2000). Wavelet Feature Vectors for Neural Network Based Harmonics Load Recognition. Proc. of the $5^{\text {th }}$ International Conference on Advances in Power System Control, Operation and Management (APSCOM), pp. 511-516.

Czarnecki, L. S. (2000). An Overview of Methods of Harmonic Suppression in Distribution Systems. Power Engineering Society Summer Meeting, pp. 800-805.
Demuth, H., Beale, M. e Hagan, M. (2007). "Neural Network Toolbox 5 - User's Guide", http: //www . mathworks. com/access / helpdesk/help/toolbox/nnet/, Acessado em 05 de Janeiro de 2008.

Dugan, R. C., McGranaghan, M. F., Santoso, S. e Beaty, H. W. (2004). Electrical Power Systems Quality, McGrawHill.

Fernandes, R. A. S., Silva, I. N., Oleskovicz, M. e Santos, F. M. C. (2008). Extração de Características Harmônicas para a Identificação de Cargas Não-Lineares Aplicando Sistemas Inteligentes. XVII Congresso Brasileiro de Automática (CBA).

Ferreira, D. D., Marques, C. A. G., Cerqueira, A. S., Duque, C. A. e Ribeiro, M. V. (2009). Sistema Automático de Detecção e Classificação de Distúrbios Elétricos em Qualidade da Energia Elétrica. Revista Controle \& Automação, vol. 20, No. 1, pp. 53-62.

Ferrero, A., Salicone, S. e Todeschini, G. (2007). A Fuzzy Method for the Identification of the Sources Reducing Harmonic Pollution in the Electric Network. Proc. of the Instrumentation and Measurement Technology Conference (IMTC), pp. 1-6.

Fluke (2005). FlukeView Power Quality Analyzer Software - User Manual.

Fluke (2006). Fluke 434/435 Three Phase Power Quality Analyzer - User Guide.

Goldschmidt, R. e Passos, E. (2005). Data Mining - Um Guia Prático, Editora Campus.

Hagan, M. T. e Menhaj, M. B. (1994). Training Feedforward Networks with the Marquardt Algorithm. IEEE Transactions on Neural Networks, vol. 5, No. 6, pp. 989993.

Hall, M. A. (1999). Correlation-based Feature Selection for Machine Learning, Ph.D. Thesis, The University of Waikato, Hamilton, New Zealand.

Hall, M. A. (2000). Correlation-based Feature Selection for Discrete and Numeric Class Machine Learning. Proc. of the $17^{\text {th }}$ International Conference on Machine Learning (ICML), pp. 359-366.

Hall, M. A. e Holmes, G. (2003). Benchmarking Attribute Selection Techniques for Discrete Class Data Mining. IEEE Transactions on Knowledge and Data Engineering, vol. 15, No. 3, pp. 1-16.

Halpin, S. M. (2005). Comparison of IEEE and IEC Harmonic standards. Power Engineering Society General Meeting, pp. 2214-2216. 
Haykin, S. (1999). Neural Networks - A Comprehensive Foundation, Prentice Hall.

Heydt, G. T. (1989). Identification of Harmonic Sources by a State Estimation Technique. IEEE Transactions on Power Delivery, vol. 4, No. 1, pp. 569-576.

IEEE Standard 519-1992 (Recommended Practices and Requirements for Harmonic Control in Electrical Power Systems).

Kohavi, R. e John, G. H. (1997). Wrappers for Feature Subset Selection. Artificial Intelligence, pp. 273-324.

Kumar, A., Das, B. e Sharma, J. (2004). Determination of location of multiple harmonic sources in a power system. Electrical Power and Energy Systems, vol. 26, pp. 73-78.

Limin, X., Weisheng, X. e Youling, Y. (2007). A Fast Harmonic Detection Method Based on Recursive DFT. Proc. of the $8^{\text {th }}$ International Conf. on Electronic Measurement and Instruments (ICEMI), pp. 972-976.

Lin, W., Lin, C., Tu, K. e Wu, C. (2005). Multiple Harmonic Source Detection and Equipment Identification With Cascade Correlation Network. IEEE Transactions on Power Delivery, vol. 20, No. 3, pp. 2166-2173.

Liu, H. e Motoda, H. (1998). Feature Selection for Knowledge Discovery and Data Mining, Kluwer Academic Publishers.

Liu, H. e Setiono, R. (1996). A Probabilistic Approach to Feature Selection: A Filter Solution. Proc. of the $13^{\text {th }}$ International Conference on Machine Learning.

Lowe, D., Waddell, R. e McGranaghan, M. (2006). Utility Perspective on Applying Harmonic Limits for Customers. Transmission and Distribution Conference and Exhibition, pp. 1152-1154.

Maitra, A., Halpin, S. M. e Litton, C. A. (2007). Applications of Harmonic Limits at Wholesale Points of Delivery. IEEE Transactions on Power Delivery, vol. 22, No. 1, pp. 263-269.

Mitzenmacher, M., e Upfal, E. (2005). Probability and Computing: Randomized Algorithms and Probabilistic Analysis, Cambridge University Press.

Mota, L. T. M., Mota, A. A. e França, A. L. M. (2004). Modelagem e Simulação de Cargas Residenciais Termostáticas para a Recomposição do Sistema Elétrico a Partir de uma Abordagem Orientada a Objetos. Revista Controle \& Automação, vol. 15, No. 2, pp. 202-214.
Nawi, S. M., Johari, J. e Abidin, A. F. (2003). A Fuzzy Logic Application for Identification of Harmonics Disturbances Sources. Proc. of the National Power and Energy Conference (PECon), pp. 27-31.

Ng, C. H., Busawon, K., Putrus, G. A. e Ran, L. (2005). FastIndividual-Harmonic-Extraction Technique. IEE Proc.Gener. Transm. Distrib., vol. 152, No. 4, pp. 556-562.

Niekerk, C. R., Rens, A. P. J. e Hoffman, A. J. (2002). Identification of Types of Distortion Sources in Power Systems by Applying Neural Networks. Proc. of the IEEE Africon, pp. 829-834.

Pádua, M. S., Lopes, A., Dechmann, S. M., Marafão, F. P. e Moreira, A. C. (2007). Metodologia para Identificação do Componente Fundamental da Tensão da Rede Baseada no Algoritmo Recursivo da TDF. Revista Controle \& Automação, vol. 18, No. 3, pp. 381-396.

Pomilio, J. A. e Deckmann, S. M. (2007). Characterization and Compensation of Harmonics and Reactive Power of Residential and Commercial Loads. IEEE Transactions on Power Delivery, vol. 22, No. 2, pp. 1049-1055.

Prudenzi, A., Grasselli, U. e Lamedica, R. (2001). IEC Std. 61000-3-2 Harmonic Current Emission Limits in Practical Systems: Need of Considering Loading Level and Attenuation Effects. Power Engineering Society General Meeting, pp. 277-282.

Quesada, I., Lázaro, A., Barrado, A., Vázquez, R., González, I. e Herreros, N. (2007). Extension of the Harmonic Elimination Technique in the Presence of Non Linear Loads. Power Electronics Specialists Conference, pp. 43-46.

Srinivasan, D., Ng, W. S. e Liew. A. C. (2006). Neural Network-Based Signature Recognition for Harmonic Source Identification. IEEE Transactions on Power Delivery, vol. 21, No. 01, pp. 398-405.

Tarasiuk, T. (2007). Hybrid Wavelet-Fourier Method for Harmonics and Harmonic Subgroups Measurement - Case Study. IEEE Transactions on Power Delivery, vol. 22, No. 1, pp. 4-17.

Tse, N. C. F. (2006). Practical Application of Wavelet to Power Quality Analysis. Power Engineering Society General Meeting, pp. 18-22.

Umeh, K. e Mohamed, A. (2005). Intelligent System for Identification of Harmonics Originating from Single Phase Nonlinear Loads. Proc. of the IEEE SoutheastCon, pp. 137-142. 
Varadan, S. e Makram, E. B. (1994). Practical Considerations in the Application of Neural Networks to the Identification of Harmonic Loads. Electrical Power Systems Research, vol. 30, pp. 103-106.

Varadan, S. e Makram, E. B. (1996). Harmonic Load Identification and Determination of Load Composition Using a Least Squares Method. Electrical Power Systems Research, vol. 37, pp. 203-208.

Variz, A. M., Jr. Carneiro, S., Pereira, J. L. R. e Barbosa, P. G. (2008). Cálculo do Fluxo de Potência de Harmônicos em Sistemas de Potência Trifásicos Utilizando o Método de Injeção de Correntes com Solução Iterativa. Revista Controle \& Automação, vol. 19, No. 2, pp.178198.

Witten, I. H. e Frank, E. (2005). Data Mining: Practical Machine Learning Tools and Techniques, Morgan Kaufmann. 